\title{
Multichannel coherence in strong-field ionization
}

\author{
Nina Rohringer ${ }^{1}$ and Robin Santra ${ }^{2,3, *}$ \\ ${ }^{1}$ Lawrence Livermore National Laboratory, Livermore, California 94551, USA \\ ${ }^{2}$ Argonne National Laboratory, Argonne, Illinois 60439, USA \\ ${ }^{3}$ Department of Physics, University of Chicago, Chicago, Illinois 60637, USA
}

(Received 24 February 2009; published 6 May 2009)

\begin{abstract}
Atomic and molecular ions generated by a strong optical laser pulse are not in general in the electronic ground state. The density matrix for such ions is characterized by the electronic quantum-state populations and by the coherences among the electronic quantum states. Nonvanishing coherences signal the presence of coherent electronic wave-packet dynamics in the laser-generated ions. For noble-gas atoms heavier than helium, the most important channels populated via strong-field ionization are the outer-valence single-hole states with a total angular momentum of $j=3 / 2$ or $j=1 / 2$. For this case, we develop a time-dependent multichannel theory of strong-field ionization. We derive the ion density matrix and express the hole density in terms of the elements of the ion density matrix. Our wave-packet calculations demonstrate that neon ions generated in a strong optical field $(800 \mathrm{~nm})$ are almost perfectly coherent. In strong-field-generated xenon ions, however, the coherence is substantially suppressed.
\end{abstract}

DOI: 10.1103/PhysRevA.79.053402

PACS number(s): $32.80 . \mathrm{Rm}, 31.15 . \mathrm{A}-$, 32.10.Fn

\section{INTRODUCTION}

The response of atoms and molecules to strong optical fields has been the subject of intense research over the last four decades. Topics studied include nonperturbative multiphoton ionization [1-16]; above-threshold ionization [17-26]; nonsequential multielectron processes [27-36]; high-harmonic generation [37-51]; attosecond physics [52-58]; and ultrafast molecular probing [59-76].

The first step in all strong-field phenomena is the ionization of an electron via nonperturbative multiphoton absorption. The tunneling picture of strong-field ionization suggests that the ion generated in a strong optical field has a hole in the orbital with the lowest binding energy. For this reason, the focus in theoretical studies has been on a single-channel description of strong-field ionization. The single-channel description is typically implemented in terms of the so-called single-active-electron approach [77-84].

Photoelectron spectroscopy reveals that in strong-field ionization of $\mathrm{Xe}$, for instance, not only the $5 p_{3 / 2}^{-1}$ ground-state manifold of the ion is populated, but also the $5 p_{1 / 2}^{-1}$ excited manifold $[85,86]$. (The notation $n l_{j}^{-1}$ indicates that, relative to the ground-state configuration of the atom, a hole is present in the $n l_{j}$ subshell.) Furthermore, the $2 j+1$ states associated with the $n l_{j}^{-1}$ configuration of a noble-gas ion are, in general, not uniformly populated: x-ray-absorption spectroscopy of laser-generated $\mathrm{Kr}$ ions demonstrated spatial alignment of the orbital hole $[87,88]$. Complete ion quantumstate populations, i.e., the diagonal elements of the ion density matrix in the ion eigenstate basis, were determined experimentally and theoretically in Ref. [89] (Xe) and Ref. [90] $(\mathrm{Kr})$.

An important question is whether the ion density matrix has any nonzero off-diagonal elements. Are there any coher-

\footnotetext{
*Corresponding author.
}

ences? The presence of coherences would imply that an ion generated by a strong optical field undergoes coherent electronic wave-packet dynamics. It is tempting to expect that in the noble-gas atoms (He excluded), the laser electric field is so strong that it will, effectively, pull an electron out of the valence $p_{z}$ orbital, where $z$ indicates the laser polarization axis. A noble-gas ion with a hole in a $p_{z}$ orbital is not in an eigenstate, but in a coherent superposition of $j=3 / 2$ and $j$ $=1 / 2$ fine-structure states. Thus, if the interaction of the laser field with the atomic electrons were stronger than the intraatomic spin-orbit interaction, essentially perfectly coherent electronic wave packets would be formed. However, using a version of the tunneling model including spin-orbit interaction [91], it was found that even at saturation, the laser electric field is not strong enough to break spin-orbit coupling in $\mathrm{Xe}$ [89] or $\mathrm{Kr}[90,91]$.

The purpose of this paper is to investigate the density matrix of an ion generated in a strong optical field using a treatment based on the numerical solution of the timedependent Schrödinger equation. We use a multichannel method that is similar to the time-dependent configurationinteraction-singles method discussed in Ref. [92]. However, we neglect electronic channel coupling and focus on the impact of the spin-orbit coupling in the ion. In Sec. II, we derive multichannel equations of motion; determine and analyze the ion density matrix; and express the hole density $[93,94]$ in terms of the ion density matrix. In Sec. III, we present numerical results for $\mathrm{Ne}$ and $\mathrm{Xe}$. Among the stable noble-gas species (He again excluded), Ne has the highest ionization potential and displays the smallest fine-structure effects; Xe has the lowest ionization potential and displays the strongest fine-structure effects. These differences have a substantial impact on the properties of the respective ion density matrix - and thus on the time evolution of the hole density. Conclusions are drawn in Sec. IV. Atomic units are used throughout, unless otherwise noted. 


\section{THEORY}

\section{A. Equations of motion}

The following discussion focuses on an atom with a closed-shell ground state. Let $\left|\varphi_{p}\right\rangle$ stand for the eigenstates (spin orbitals) of the selected one-particle Hamiltonian $\hat{h}_{0}$ (the index $p$ denotes spatial and spin degrees of freedom), i.e.,

$$
\hat{h}_{0}\left|\varphi_{p}\right\rangle=\varepsilon_{p}\left|\varphi_{p}\right\rangle \text {. }
$$

The $\varepsilon_{p}$ are the orbital energies. In the language of second quantization [95,96], the laser-free ground state of an $N$-electron closed-shell system may be written as

$$
\left|\Phi_{0}\right\rangle=\prod_{i=1}^{N} \hat{c}_{i}^{\dagger}|0\rangle,
$$

where $|0\rangle$ is the vacuum state and $\hat{c}_{p}^{\dagger}$ creates an electron in spin orbital $\left|\varphi_{p}\right\rangle$, i.e., $\hat{c}_{p}^{\dagger}|0\rangle=\left|\varphi_{p}\right\rangle$. The one-particle Hamiltonian in the absence of the laser field reads

$$
\hat{H}_{0}=\sum_{p, q}\left\langle\varphi_{p}\left|\hat{h}_{0}\right| \varphi_{q}\right\rangle \hat{c}_{p}^{\dagger} \hat{c}_{q}=\sum_{p} \varepsilon_{p} \hat{c}_{p}^{\dagger} \hat{c}_{p},
$$

so that

$$
\hat{H}_{0}\left|\Phi_{0}\right\rangle=\left\{\sum_{i} \varepsilon_{i}\right\}\left|\Phi_{0}\right\rangle .
$$

Here and in the following, indices $i, j, k, l, \ldots$ are used for spin orbitals that are occupied in $\left|\Phi_{0}\right\rangle$. Unoccupied (virtual) orbitals are symbolized by indices $a, b, c, d, \ldots$, whereas for general orbitals (occupied or unoccupied) indices $p, q, r, s, \ldots$ are employed.

Let the $N$-electron system be subject to a laser field [field strength $\mathcal{E}(t)]$ linearly polarized along the $z$ axis. Within the electric dipole approximation, the time-dependent Hamiltonian can be written as

$$
\hat{H}(t)=\hat{H}_{0}-E_{0}-\mathcal{E}(t) \hat{Z}-i \eta \hat{W} .
$$

A constant energy shift has been introduced to render subsequent equations more compact. The energy shift is chosen as the ground-state energy of the $N$-electron system [cf. Eq. (4)]:

$$
E_{0}=\sum_{i} \varepsilon_{i}
$$

The dipole operator, shown here in the length form, is a one-body operator,

$$
\hat{Z}=\sum_{p q} z_{p q} \hat{c}_{p}^{\dagger} \hat{c}_{q},
$$

where the dipole matrix elements in terms of spin orbitals (two-component spinors) are defined as

$$
z_{p q}=\int d^{3} x \boldsymbol{\varphi}_{p}^{\dagger}(\boldsymbol{x}) z \varphi_{q}(\boldsymbol{x}) .
$$

The last operator in Eq. (5), $-i \eta \hat{W}=-i \eta \sum_{p q} w_{p q} \hat{c}_{p}^{\dagger} \hat{c}_{q}$, is a complex absorbing potential (CAP) [97-101]. The CAP ab- sorbs the strong-field-ionized electron and renders the associated wave function square integrable. The real, nonnegative parameter $\eta$ is the CAP strength. The local oneelectron potential $w(r)$ is chosen here as

$$
w(r)= \begin{cases}0, & 0 \leq r<c \\ (r-c)^{2}, & r \geq c .\end{cases}
$$

Inside a sphere of radius $c$, the CAP vanishes. The sphere must be chosen large enough such that electron trajectories leading to electron-ion recollision remain unperturbed. Thus, the CAP acts only near the end of the radial grid. In particular, we can ensure that CAP matrix elements involving one or more occupied orbitals vanish, i.e., $w_{a i}=0, w_{i a}=0$, and $w_{i i^{\prime}}=0$. This is exploited in the following.

The time evolution of the $\mathrm{N}$-electron system under the influence of the laser field is governed by the time-dependent Schrödinger equation,

$$
i \frac{\partial}{\partial t}|\Psi, t\rangle=\hat{H}(t)|\Psi, t\rangle .
$$

The laser field couples the ground state $\left|\Phi_{0}\right\rangle$ to excited-state configurations. Taking into account only single excitations with respect to $\left|\Phi_{0}\right\rangle$, the many-electron wave packet may be written as

$$
|\Psi, t\rangle=\alpha_{0}(t)\left|\Phi_{0}\right\rangle+\sum_{i} \sum_{a} \alpha_{i}^{a}(t) e^{-i\left(\varepsilon_{a}-\varepsilon_{i}\right) t}\left|\Phi_{i}^{a}\right\rangle .
$$

The basis vector

$$
\left|\Phi_{i}^{a}\right\rangle=\hat{c}_{a}^{\dagger} \hat{c}_{i}\left|\Phi_{0}\right\rangle
$$

describes the excitation of an electron from $\left|\varphi_{i}\right\rangle$ to $\left|\varphi_{a}\right\rangle$. The assumption made here is that the laser field does not excite more than one of the initially occupied orbitals. This is a single-active-electron picture allowing for the inclusion of several one-hole ionization channels.

Assuming that before the laser pulse the atom is in its ground state, the expansion coefficients $\alpha_{0}(t)$ and $\alpha_{i}^{a}(t)$ in Eq. (11) must satisfy the initial conditions

$$
\begin{aligned}
& \alpha_{0}(t \rightarrow-\infty)=1, \\
& \alpha_{i}^{a}(t \rightarrow-\infty)=0 .
\end{aligned}
$$

Inserting Eq. (11) into Eq. (10), projecting the resulting expressions onto $\left|\Phi_{0}\right\rangle$ and $\left|\Phi_{i}^{a}\right\rangle$, respectively, and exploiting the rules for evaluating matrix elements with respect to Slater determinants [102], the equations of motion for the expansion coefficients $\alpha_{0}$ and $\alpha_{i}^{a}$ can be derived:

$$
\begin{gathered}
i \dot{\alpha}_{0}=-\mathcal{E}(t) \sum_{a, i} \alpha_{i}^{a} e^{-i\left(\varepsilon_{a}-\varepsilon_{i}\right) t} z_{i a}, \\
i \dot{\alpha}_{i}^{a}=-\mathcal{E}(t) \alpha_{0} e^{i\left(\varepsilon_{a}-\varepsilon_{i}\right) t} z_{a i}-\mathcal{E}(t) \sum_{a^{\prime}} \alpha_{i}^{a^{\prime}} e^{i\left(\varepsilon_{a}-\varepsilon_{a^{\prime}}\right) t} z_{a a^{\prime}} \\
-i \eta \sum_{a^{\prime}} \alpha_{i}^{a^{\prime}} e^{i\left(\varepsilon_{a}-\varepsilon_{a^{\prime}}\right) t} w_{a a^{\prime}} .
\end{gathered}
$$

Diagonal dipole terms $z_{i i}$ and $z_{a a}$ vanish for atomic systems 
and have been omitted. Also not shown are matrix elements of the form $z_{i i}$, since we are interested here in ionization from the outer valence shell only. All spin orbitals associated with the outer valence shell have the same parity (fixed $l$ ), so that $z_{i i^{\prime}}=0$.

\section{B. Ion density matrix}

An important quantity that may be calculated within this framework is the reduced density matrix of the residual ion produced in the excitation (ionization) process. Let

$$
\hat{\rho}(t)=|\Psi, t\rangle\langle\Psi, t|
$$

denote the density operator of the $N$-electron system. It follows from Eqs. (5) and (10) that, because of the CAP, $\operatorname{tr}[\hat{\rho}]$ $=\langle\Psi, t \mid \Psi, t\rangle$ is not conserved:

$$
\frac{d}{d t}\langle\Psi, t \mid \Psi, t\rangle=-2 \eta \sum_{a, a^{\prime}, i} \alpha_{i}^{a *} \alpha_{i}^{a^{\prime}} e^{i\left(\varepsilon_{a}-\varepsilon_{a^{\prime}}\right) t} w_{a a^{\prime}} .
$$

Therefore, care must be taken in order to construct an ion density matrix with a norm that remains conserved after the laser pulse. If we followed the standard strategy in Hermitian quantum mechanics [103], matrix elements of the reduced density matrix of the residual ion would be defined as

$$
\widetilde{\rho}_{i i^{\prime}}^{(\text {ion })}(t)=\sum_{a}\left\langle\Phi_{i}^{a}|\hat{\rho}(t)| \Phi_{i^{\prime}}^{a}\right\rangle=e^{i\left(\varepsilon_{i}-\varepsilon_{i^{\prime}}\right) t} \sum_{a} \alpha_{i}^{a} \alpha_{i^{\prime}}^{a *} .
$$

After the laser pulse, the time dependence of the ion densitymatrix element should be fully contained in the factor $\exp \left[i\left(\varepsilon_{i}-\varepsilon_{i^{\prime}}\right) t\right]$. In other words, the ion density matrix in the interaction picture should be stationary for sufficiently large $t$. Using Eq. (16), one may derive an equation of motion for $\Sigma_{a} \alpha_{i}^{a} \alpha_{i^{\prime}}^{a *}$. From this it follows that the correct ion density matrix in the presence of the CAP is given by

$$
\begin{aligned}
\rho_{i i^{\prime}}^{(\mathrm{ion})}(t)= & e^{i\left(\varepsilon_{i}-\varepsilon_{i^{\prime}}\right) t}\left\{\sum_{a} \alpha_{i}^{a}(t) \alpha_{i^{\prime}}^{a *}(t)\right. \\
& \left.+2 \eta \int_{-\infty}^{t} d t^{\prime} \sum_{a, a^{\prime}} \alpha_{i}^{a^{\prime}}\left(t^{\prime}\right) \alpha_{i^{\prime}}^{a *}\left(t^{\prime}\right) e^{i\left(\varepsilon_{a}-\varepsilon_{a^{\prime}}\right) t^{\prime}} w_{a a^{\prime}}\right\} .
\end{aligned}
$$

A formally equivalent expression obtained from the equation of motion for $\Sigma_{a} \alpha_{i}^{a} \alpha_{i^{\prime}}^{a *}$ is

$$
\begin{aligned}
\rho_{i i^{\prime}}^{(\text {ion })}(t)= & e^{i\left(\varepsilon_{i}-\varepsilon_{i^{\prime}}\right) t} \int_{-\infty}^{t} d t^{\prime} \frac{\mathcal{E}\left(t^{\prime}\right)}{i}\left\{\alpha_{0}^{*}\left(t^{\prime}\right) \sum_{a} \alpha_{i}^{a}\left(t^{\prime}\right) e^{i\left(\varepsilon_{i^{\prime}}-\varepsilon_{a}\right) t^{\prime}} z_{i^{\prime} a}\right. \\
& \left.-\alpha_{0}\left(t^{\prime}\right) \sum_{a} \alpha_{i^{\prime}}^{a *}\left(t^{\prime}\right) e^{i\left(\varepsilon_{a}-\varepsilon_{i}\right) t^{\prime}} z_{a i}\right\}
\end{aligned}
$$

This shows explicitly that the ion density matrix becomes stationary after the laser pulse. In addition, since Eq. (21) does not depend on a double sum over virtual orbitals, it is computationally more advantageous than Eq. (20).

The ion density matrix can be used to explore whetherand to which degree-the strong-field ionization process establishes coherences among the one-hole ionization chan- nels. With the help of Eqs. (13)-(15) and (21), we find that

$$
\left|\alpha_{0}(t)\right|^{2}+\sum_{i} \rho_{i i}^{(\text {ion })}(t)=1
$$

Thus, the diagonal element $\rho_{i i}^{(\text {ion })}(t)$ may be interpreted as the probability of forming a hole in orbital $\left|\varphi_{i}\right\rangle$.

\section{Atomic Hamiltonian}

We employ the atomic one-electron Hamiltonian

$$
\hat{h}_{0}=-\frac{1}{2} \nabla^{2}+V_{\mathrm{HS}}(r)+\frac{1}{2} \alpha^{2} \frac{1}{r} \frac{d V_{\mathrm{HS}}}{d r} \boldsymbol{l} \cdot \boldsymbol{s} .
$$

The effective one-electron potential $V_{\mathrm{HS}}$ is calculated using the Hartree-Fock-Slater $[104,105]$ code written by Herman and Skillman [106]. $V_{\mathrm{HS}}$ describes the Coulomb interaction of an electron with the nucleus and with the mean field generated by the other electrons [107-110]. For a neutral atom of nuclear charge $+Z, V_{\mathrm{HS}}$ satisfies

$$
V_{\mathrm{HS}}(r) \rightarrow-Z / r, \quad r \rightarrow 0,
$$

and

$$
V_{\mathrm{HS}}(r) \rightarrow-1 / r, \quad r \rightarrow \infty .
$$

The operator in Eq. (23) depending on the scalar product of the electron's orbital angular momentum $l$ and spin $s$ describes the spin-orbit interaction [111] ( $\alpha$ is the fine-structure constant). It will be assumed that the spin-orbit interaction affects only occupied orbitals. This means that the ionization potential $I_{i}=-\varepsilon_{i}$ associated with the production of a hole in $\left|\varphi_{i}\right\rangle$ reflects the fine structure of the ionic eigenstates. The dynamics of the excited electron is assumed to be unaffected by the spin-orbit interaction. This allows us to reduce the complexity of the wave-packet propagation problem.

It should be pointed out that if we wanted to include spinorbit coupling for the virtual orbitals, we should, in principle, also take into consideration the tensorial interaction between the excited electron and the ion core. (A noble-gas ion with an $n p^{-1}$ configuration has an electric quadrupole moment in its ${ }^{2} P_{3 / 2}$ states, but not in its ${ }^{2} P_{1 / 2}$ states.) The analysis of the $n p^{-1} n^{\prime} p$ configuration presented in the book by Condon and Shortley [112] suggests that the anisotropic contributions to the electrostatic interaction between the excited electron and the ion core are as important as spin-orbit coupling for the virtual orbitals. Both effects are relatively small and are neglected here.

In practice, we solve the radial eigenvalue problem

$$
\left\{-\frac{1}{2} \frac{d^{2}}{d r^{2}}+\frac{l(l+1)}{2 r^{2}}+V_{\mathrm{HS}}(r)\right\} u_{n, l}(r)=E_{n, l} u_{n, l}(r),
$$

subject to the boundary conditions $u_{n, l}(0)=0$ and $u_{n, l}\left(r_{\max }\right)$ $=0$, where $r=r_{\max }$ signals the end of the radial grid employed in the numerical calculation. Thus, for the virtual orbitals $\varphi_{a}(\boldsymbol{x})=\varphi_{n, l, m_{l}, m_{s}}^{(v)}(\boldsymbol{x})$ we have 


$$
\varphi_{n, l, m_{l}, m_{s}}^{(v)}(\boldsymbol{x})=\frac{u_{n, l}^{(v)}(r)}{r} Y_{l, m_{l}}(\theta, \phi)\left(\begin{array}{c}
\delta_{m_{s}, 1 / 2} \\
\delta_{m_{s}, 1 / 2}
\end{array}\right) .
$$

Here, $Y_{l, m_{l}}(\theta, \phi)$ is a spherical harmonic. The energies of virtual orbitals are taken directly from Eq. (26); i.e., we set $\varepsilon_{a}=\varepsilon_{n, l}^{(v)}=E_{n, l}^{(v)}$. Treating spin-orbit coupling within the $n, l$ subshell in the standard way using degenerate-state perturbation theory, the occupied orbitals $\varphi_{i}(x)=\varphi_{n, l, j, m}^{(o)}(x)$ are given by

$$
\varphi_{n, l, j, m}^{(o)}(\boldsymbol{x})=\frac{u_{n, l}^{(o)}(r)}{r}\left(\begin{array}{c}
C(l, s, j ; m-1 / 2,1 / 2, m) Y_{l, m-1 / 2}(\theta, \phi) \\
C(l, s, j ; m+1 / 2,-1 / 2, m) Y_{l, m+1 / 2}(\theta, \phi)
\end{array}\right) .
$$

In this expression, $C\left(l, s, j ; m_{l}, m_{s}, m\right)(s=1 / 2)$ is a ClebschGordan coefficient [113]. For the energies of occupied orbitals, we take experimental ionization potentials $I_{n, l, j}$ and set $\varepsilon_{i}=\varepsilon_{n, l, j}^{(o)}=-I_{n, l, j}$.

\section{Symmetry analysis}

The matrix elements needed for the evaluation of Eqs. (15) and (16) are now easily determined:

$$
\begin{aligned}
z_{a i}= & \left\langle\varphi_{n, l, m_{l}, m_{s}}^{(v)}|z| \varphi_{n^{\prime}, l^{\prime}, j, m}^{(o)}\right\rangle=C\left(l^{\prime}, s, j ; m_{l}, m_{s}, m\right) \\
& \times \sqrt{\frac{2 l^{\prime}+1}{2 l+1}} C\left(l^{\prime}, 1, l ; m_{l}, 0, m_{l}\right) C\left(l^{\prime}, 1, l ; 0,0,0\right) \\
& \times \int_{0}^{r_{\max }} d r u_{n, l}^{(v)}(r) r u_{n^{\prime}, l^{\prime}}^{(o)}(r), \\
z_{a a^{\prime}}= & \left\langle\varphi_{n, l, m_{l}, m_{s}}^{(v)}|z| \varphi_{n^{\prime}, l^{\prime}, m_{l}^{\prime}, m_{s}^{\prime}}^{(v)}\right\rangle=\delta_{m_{l}, m_{l}^{\prime}} \delta_{m_{s^{\prime}}, m_{s}^{\prime}} \\
& \times \sqrt{\frac{2 l^{\prime}+1}{2 l+1}} C\left(l^{\prime}, 1, l ; m_{l}, 0, m_{l}\right) C\left(l^{\prime}, 1, l ; 0,0,0\right) \\
& \times \int_{0}^{r_{\max }} d r u_{n, l}^{(v)}(r) r u_{n^{\prime}, l^{\prime}}^{(v)}(r),
\end{aligned}
$$

and

$$
\begin{aligned}
w_{a a^{\prime}}= & \left\langle\varphi_{n, l, m_{l}, m_{s}}^{(v)}|w| \varphi_{n^{\prime}, l^{\prime}, m_{l}^{\prime}, m_{s}^{\prime}}^{(v)}\right\rangle=\delta_{m_{l}, m_{l}^{\prime}} \delta_{m_{s}, m_{s}^{\prime}} \delta_{l, l^{\prime}} \\
& \times \int_{0}^{r_{\max }} d r u_{n, l}^{(v)}(r) w(r) u_{n^{\prime}, l}^{(v)}(r) .
\end{aligned}
$$

We focus on active occupied orbitals $\varphi_{n, l, j, m}^{(o)}(x)$ with fixed $n=n_{o}$ and $l=l_{o}$. Only $j$ and $m$ are allowed to vary. In the following we employ the notation $\alpha_{i}^{a}=\alpha_{n_{o}, l_{o}, j, m}^{n, l, m_{s}, m_{s}}$. Hence, Eqs. (15) and (16) may be written as

$$
\begin{aligned}
i \dot{\alpha}_{0}= & -\mathcal{E}(t) \sum_{j, m} \sum_{n, l, m_{l}, m_{s}} \alpha_{n_{o}, l_{o}, j, m}^{n, l, m_{l}, m_{s}} e^{-i\left(E_{n, l}^{(v)}+I_{n_{o}, l_{o}, j}\right) t} \\
& \times\left\langle\varphi_{n_{o}, l_{o}, j, m}^{(o)}|z| \varphi_{n, l, m_{l}, m_{s}}^{(v)}\right\rangle
\end{aligned}
$$

and
TABLE I. Classes of excited-state expansion coefficients $\alpha_{n_{o}, l_{o}, j, m}^{n, l, m_{l}, m_{s}}$ required for constructing the $N$-electron wave function $|\Psi, t\rangle$

\begin{tabular}{cccc}
\hline \hline$j$ & $m$ & $m_{l}$ & $m_{s}$ \\
\hline $3 / 2$ & $+3 / 2$ & +1 & $+1 / 2$ \\
$3 / 2$ & $+1 / 2$ & +1 & $-1 / 2$ \\
$3 / 2$ & $+1 / 2$ & 0 & $+1 / 2$ \\
$1 / 2$ & $+1 / 2$ & +1 & $-1 / 2$ \\
$1 / 2$ & $+1 / 2$ & 0 & $+1 / 2$ \\
\hline \hline
\end{tabular}

$$
\begin{aligned}
i \dot{\alpha}_{n_{o}, l_{o}, j, m}^{n, l, m_{l}, m_{s}}= & -\mathcal{E}(t) \alpha_{0} e^{i\left(E_{n, l}^{(v)+I_{n_{o}}, l_{o}, j}\right) t} \times\left\langle\varphi_{n, l, m_{l}, m_{s}}^{(v)}|z| \varphi_{n_{o}, l_{o}, j, m}^{(o)}\right\rangle \\
& -\mathcal{E}(t) \sum_{n^{\prime}, l^{\prime}} \alpha_{n_{o}, l_{o}, j, m}^{n^{\prime}, l^{\prime}, m_{l}, m_{s}} e^{i\left(E_{n, l}^{(v)}-E_{n^{\prime}, l^{\prime}}^{(v)}\right) t} \\
& \times\left\langle\varphi_{n, l, m_{l}, m_{s}}^{(v)}|z| \varphi_{n^{\prime}, l^{\prime}, m_{l}, m_{s}}^{(v)}\right\rangle \\
& -i \eta \sum_{n^{\prime}} \alpha_{n_{o}, l_{o}, j, m}^{n^{\prime}, l, m_{l}, m_{s}} e^{i\left(E_{n, l}^{(v)}-E_{n^{\prime}, l}^{(v)}\right) t} \\
& \times\left\langle\varphi_{n, l, m_{l}, m_{s}}^{(v)}|w| \varphi_{n^{\prime}, l, m_{l}, m_{s}}^{(v)}\right\rangle .
\end{aligned}
$$

From Eqs. (13), (14), (29), and (33) it follows that $\alpha_{n_{o}, l_{o}, j, m}^{n, l, m_{l}, m_{s}}$ $=0$ unless $m_{l}+m_{s}=m$. As a consequence, the ion density matrix [cf. Eqs. (20) and (21)] is diagonal with respect to $m$. We therefore write the elements of the ion density matrix as $\rho_{j, j^{\prime}}^{(m)}$ ( $n_{o}$ and $l_{o}$ are fixed).

We may further conclude from Eqs. (13), (14), (29), and (33) that

$$
\alpha_{n_{o}, l_{o}, j,-m}^{n, l,-m l_{l},-m_{s}}=(-1)^{l_{o}+s-j} \alpha_{n_{o}, l_{o}, j, m}^{n, l, m_{l}, m_{s}} .
$$

Using Eqs. (20) and (34), we find that the ion density matrix satisfies the following symmetry property:

$$
\boldsymbol{\rho}_{j, j^{\prime}}^{(-m)}=(-1)^{j-j^{\prime}} \boldsymbol{\rho}_{j, j^{\prime}}^{(m)} .
$$

We are interested here in ionization from the outer valence shell of noble-gas atoms such as $\mathrm{Ne}$ or $\mathrm{Xe}$, so that $l_{o}$ $=1$. Thus, exploiting Eq. (34), there are five classes of excited-state expansion coefficients that have to be calculated. These are listed in Table I.

\section{E. Hole density}

Let

$$
\rho_{0}(\boldsymbol{x})=\left\langle\Phi_{0}\left|\hat{\psi}^{\dagger}(\boldsymbol{x}) \hat{\psi}(\boldsymbol{x})\right| \Phi_{0}\right\rangle=\sum_{i} \varphi_{i}^{\dagger}(\boldsymbol{x}) \varphi_{i}(\boldsymbol{x})
$$

denote the electron density of the neutral atom in its ground state. In Eq. (36),

$$
\hat{\psi}(\boldsymbol{x})=\sum_{p} \varphi_{p}(\boldsymbol{x}) \hat{c}_{p}
$$

and 


$$
\hat{\psi}^{\dagger}(\boldsymbol{x})=\sum_{p} \varphi_{p}^{\dagger}(\boldsymbol{x}) \hat{c}_{p}^{\dagger}
$$

are field operators; $\hat{\psi}^{\dagger}(\boldsymbol{x}) \hat{\psi}(\boldsymbol{x})$ is the electron-density operator $[95,96]$. Employing the reduced density operator of the ion,

$$
\hat{\rho}^{(\text {ion })}(t)=\sum_{i, i^{\prime}} \hat{c}_{i}\left|\Phi_{0}\right\rangle \rho_{i i^{\prime}}^{(\text {ion })}(t)\left\langle\Phi_{0}\right| \hat{c}_{i^{\prime}}^{\dagger},
$$

the time-dependent electron density of the laser-generated ion is obtained as

$$
\begin{aligned}
\rho^{(\mathrm{ion})}(\boldsymbol{x}, t)= & \operatorname{Tr}\left[\hat{\boldsymbol{\rho}}^{(\mathrm{ion})}(t) \hat{\psi}^{\dagger}(\boldsymbol{x}) \hat{\psi}(\boldsymbol{x})\right] \\
= & \sum_{i} \rho_{i i}^{(\mathrm{ion})}(t) \sum_{i^{\prime}} \varphi_{i^{\prime}}^{\dagger}(\boldsymbol{x}) \varphi_{i^{\prime}}(\boldsymbol{x}) \\
& -\sum_{i, i^{\prime}} \rho_{i i^{\prime}}^{(\mathrm{ion})}(t) \varphi_{i}^{\dagger}(\boldsymbol{x}) \varphi_{i^{\prime}}(\boldsymbol{x}) .
\end{aligned}
$$

We thus define the hole density as

$$
\begin{aligned}
Q(\boldsymbol{x}, t) & =\left[1-\left|\alpha_{0}(t)\right|^{2}\right] \rho_{0}(\boldsymbol{x})-\rho^{(\mathrm{ion})}(\boldsymbol{x}, t) \\
& =\sum_{i, i^{\prime}} \rho_{i i^{\prime}}^{(\mathrm{ion})}(t) \varphi_{i}^{\dagger}(\boldsymbol{x}) \varphi_{i^{\prime}}(\boldsymbol{x}),
\end{aligned}
$$

where we made use of Eqs. (22), (36), and (40). The total hole charge is

$$
\int d^{3} x Q(\boldsymbol{x}, t)=1-\left|\alpha_{0}(t)\right|^{2},
$$

i.e., it equals the total excitation (ionization) probability. Equation (41) generalizes the definition of the hole density given in Refs. [93,94]. The definition employed here takes into consideration the formation of the hole. Moreover, Eq. (41) anticipates that the ionization by the strong laser field may not necessarily be completely saturated. In the case that ionization is saturated $\left[\alpha_{0}(t)=0\right]$, our definition is identical to the one adopted in Refs. [93,94].

Considering the formation of a hole in an occupied $n p$ subshell (the outer valence shell of $\mathrm{Ne}$ or $\mathrm{Xe}$, for instance), the hole density [Eq. (41)] may be written as

$$
\begin{aligned}
Q(x, t)= & \rho_{n, 1}^{(o)}(r)\left[\frac{1}{2} \rho_{3 / 2,3 / 2}^{(3 / 2)}(t) \sin ^{2} \theta+\frac{1}{2} \rho_{3 / 2,3 / 2}^{(1 / 2)}(t)\left(\cos ^{2} \theta+\frac{1}{3}\right)\right. \\
& \left.+\frac{1}{3} \rho_{1 / 2,1 / 2}^{(1 / 2)}(t)+\sqrt{2} \operatorname{Re}\left\{\rho_{3 / 2,1 / 2}^{(1 / 2)}(t)\right\}\left(\frac{1}{3}-\cos ^{2} \theta\right)\right],
\end{aligned}
$$

where we exploited the properties of the ion density matrix (cf. Sec. II D) and the definition of the spherical harmonics. In Eq. (43), we introduced the total electron density in the closed $n p$ subshell of the neutral atom in its ground state,

$$
\rho_{n, 1}^{(o)}(r)=\frac{3}{2 \pi}\left[\frac{u_{n, 1}^{(o)}(r)}{r}\right]^{2} .
$$

The angle $\theta$ in Eq. (43) is the polar angle with respect to the laser polarization axis. The hole density is azimuthally symmetric.

\section{CALCULATIONS}

\section{A. Numerical implementation}

We typically require angular momentum quantum numbers up to $l=30$ in order to get converged results for the ion density matrix. The radial eigenfunctions $u_{n, l}(r)$ for a given $l$ [see Eq. (26)] are calculated by a generalized pseudospectral method [114-117]. In this method, the radial interval $\left[0, r_{\max }\right]$ is mapped onto the interval $[-1,1]$ by the mapping function $[114,116]$

$$
r(x)=L \frac{1+x}{1-x+\zeta},
$$

where $\zeta=2 L / r_{\max }$. The grid points $x$ of the mapped domain are chosen as the collocation points of a Gauss-Lobatto quadrature. Thereby, a nonequidistant mesh spacing is achieved. By varying the parameter $L$, the point density near the origin can be altered, i.e., a higher mesh-point density near the Coulomb singularity at the origin can be achieved, while still having enough grid points available to describe the long-range part of the Coulomb potential. We obtain convergence for the ion density matrix of $\mathrm{Ne}$ using 700 radial grid points, $r_{\max }=120$, and $\zeta=31$; for Xe we use 1000 radial grid points, $r_{\max }=200$, and $\zeta=35$. The CAP strength in our calculations is $\eta=10^{-5}$. The parameter $c$ in Eq. (9) is 80 in the case of $\mathrm{Ne}(c=150$ for $\mathrm{Xe})$.

The multichannel wave function is propagated in time employing the second-order finite difference scheme

$$
\begin{aligned}
\alpha_{n_{o}, l_{o}, j, m}^{n, l, m_{l}, m_{s}}(t+\Delta t)= & \alpha_{n_{o}, l_{o}, j, m}^{n, l, m_{l}, m_{s}}(t-\Delta t) \\
& -2 i \Delta t f\left[\alpha_{0}(t), \alpha_{n_{o}, l_{o}, j, m}^{n^{\prime}, l^{\prime}, m_{l}, m_{s}}(t)\right],
\end{aligned}
$$

where $f[\cdots]$ denotes the right-hand side of Eq. (33). The same strategy is applied to the propagation of the groundstate coefficient:

$$
\alpha_{0}(t+\Delta t)=\alpha_{0}(t-\Delta t)-2 i \Delta \alpha_{0}(t),
$$

where $\Delta \alpha_{0}(t)$ stands for the right-hand side of Eq. (32). The size of the time step is $\Delta t=0.00095$ a.u.

We keep track of the norm of the $N$-electron wave packet $|\Psi, t\rangle$ by applying the second-order finite difference scheme to Eq. (18). In order to improve the numerical stability of our wave-packet propagation calculation, we renormalize the $\mathrm{N}$-electron wave packet after each time step using the norm obtained from Eq. (18). We note that for the numerical parameters employed here, Eqs. (20) and (21) for the calculation of the ion density matrix give virtually identical results.

\section{B. Ion density matrix}

Figure 1 shows calculated ion density matrix data for $\mathrm{Ne}$ exposed to an 800-nm four-cycle laser pulse. The laser electric field is illustrated in Fig. 1(b). (A Gaussian pulse envelope gives qualitatively similar results, which are not shown here.) For our calculation on neon, we assume a field amplitude of 0.245 a.u. Before the laser pulse, the atom is assumed to reside in the electronic ground state. The time evolution of the ion quantum-state populations $\rho_{j, j}^{(m)}+\rho_{j, j}^{(-m)}=2 \rho_{j, j}^{(m)}[\mathrm{cf}$. Eq. 


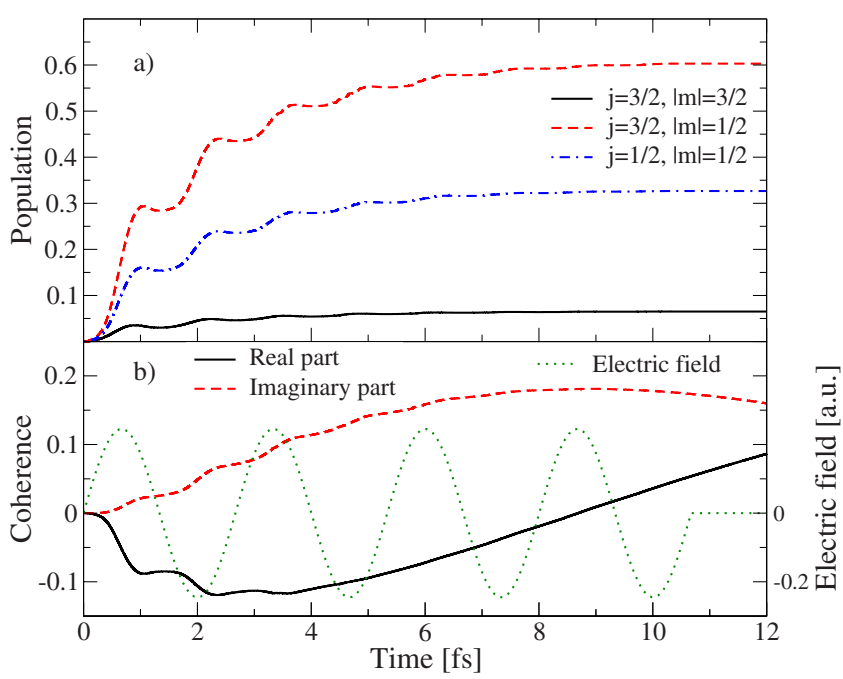

FIG. 1. (Color online) Time evolutions of the ion density-matrix elements of neon in the presence of an $800-\mathrm{nm}$ four-cycle laser pulse. The laser electric field amplitude is 0.245 a.u.

(35)] is plotted in Fig. 1(a). By the time the laser pulse is over, the sum over the ion quantum-state populations is unity; i.e., the neutral ground-state population is zero. The $j=3 / 2$ level is highly aligned. The population in the $j=3 / 2$, $m= \pm 1 / 2$ states is about $60 \%$ and is much higher than the population in the $j=3 / 2, m= \pm 3 / 2$ states. About a third of the total population is in the $j=1 / 2, m= \pm 1 / 2$ states.

In view of the symmetry properties of the ion density matrix (see Sec. II D), there can be only four nonzero offdiagonal matrix elements:

$$
\rho_{3 / 2,1 / 2}^{(1 / 2)}=\left(\rho_{1 / 2,3 / 2}^{(1 / 2)}\right)^{*}=-\rho_{3 / 2,1 / 2}^{(-1 / 2)}=-\left(\rho_{1 / 2,3 / 2}^{(-1 / 2)}\right)^{*} .
$$

In the following we simply refer to $\rho_{3 / 2,1 / 2}^{(1 / 2)}$ as the coherence. For a pure state,

$$
\left|\rho_{3 / 2,1 / 2}^{(1 / 2)}\right|=\sqrt{\rho_{3 / 2,3 / 2}^{(1 / 2)} \rho_{1 / 2,1 / 2}^{(1 / 2)}} .
$$

Hence, the maximum degree of coherence we may expect, based on the populations in Fig. $1(\mathrm{a})$, is $\left|\rho_{3 / 2,1 / 2}^{(1 / 2)}\right|=0.22$. The actual time evolutions of the real and imaginary parts of the coherence are shown in Fig. 1(b). We find that at the end of the laser pulse $\left|\rho_{3 / 2,1 / 2}^{(1 / 2)}\right|=0.18$, which is quite close to the maximum value.

The calculated ion quantum-state populations and coherence for Xe are displayed in Fig. 2. As in the case of neon, an 800-nm four-cycle laser pulse is assumed [see Fig. 2(b)]. Our calculation on xenon assumes a field amplitude of 0.09 a.u., which is chosen such that the time evolution of the total ion yield is similar to the total ion yield for neon at a laser field amplitude of 0.245 a.u. This corresponds to the matched field amplitude conditions defined in Ref. [48]. In the case of Ne, the electron binding energy for the $j=3 / 2$ channel is $21.6 \mathrm{eV}$ [118]; for $\mathrm{Xe}$, the electron binding energy for the $j=3 / 2$ channel is $12.1 \mathrm{eV}$ [119]. Therefore, the field amplitude required to saturate ionization of $\mathrm{Xe}$ is lower than for $\mathrm{Ne}$.

As may be seen in Fig. 2(a), the $j=3 / 2$ level of $\mathrm{Xe}^{+}$is strongly aligned along the laser polarization axis. This is qualitatively similar to neon. The population of the $j=1 / 2$

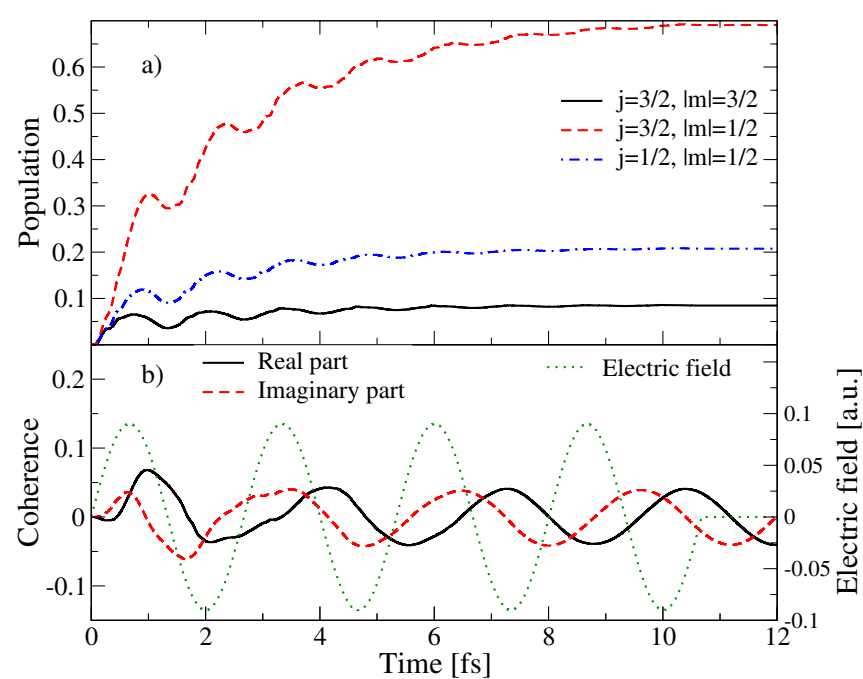

FIG. 2. (Color online) Time evolutions of the ion density-matrix elements of xenon in the presence of an 800-nm four-cycle laser pulse. The laser electric field amplitude is 0.09 a.u.

level of $\mathrm{Xe}^{+}$is smaller than the population of the $j=1 / 2$ level of $\mathrm{Ne}^{+}$. This is easily understood: in $\mathrm{Ne}^{+}$the $j=1 / 2$ level is only $0.1 \mathrm{eV}$ higher than the $j=3 / 2$ level [120], whereas in $\mathrm{Xe}^{+}$the $j=1 / 2$ level is $1.3 \mathrm{eV}$ higher than the $j=3 / 2$ level [121]. In addition, under matched field amplitude conditions, the laser electric field amplitude for $\mathrm{Ne}$ is higher than for $\mathrm{Xe}$.

Using Eq. (49) in combination with the ion quantum-state populations in Fig. 2(a), we expect that the maximum degree of coherence is $\left|\rho_{3 / 2,1 / 2}\right|=0.19$. This maximum value is quite similar to what we obtained for neon. However, it may be concluded from the real and imaginary parts of the coherence shown in Fig. 2(b) that at the end of the laser pulse $\left|\rho_{3 / 2,1 / 2}^{(1 / 2)}\right|=0.04$. The coherence in the laser-generated Xe ion is thus suppressed by almost a factor of 5 .

\section{Hole density}

The ion quantum-state populations at the end of the laser pulse [Figs. 1(a) and 2(a)] imply that generally the hole densities of $\mathrm{Ne}^{+}$and $\mathrm{Xe}^{+}$, respectively, are not spherically symmetric. The fact that there is a nonzero coherence [Figs. 1(b) and 2(b)] means that the hole density is not stationary. The laser-generated ions undergo electronic wave-packet dynamics driven by spin-orbit coupling. This is illustrated in Figs. 3 and 4 for $\mathrm{Ne}^{+}$and $\mathrm{Xe}^{+}$, respectively.

We exploit that the ratio between the hole density $Q(\boldsymbol{x}, t)$ $\left[\right.$ Eq. (43)] and the electron density $\rho_{n, 1}^{(o)}(r)[$ Eq. (44)] depends only on the polar angle $\theta$ with respect to the laser polarization axis. In Figs. 3 and 4, we show this ratio in a polar plot as a function of $\theta$. The vertical axis in these figures corresponds to the laser polarization axis.

The spin-orbit period $T^{(\mathrm{SO})}$ in $\mathrm{Ne}^{+}$is $42.7 \mathrm{fs} ; T^{(\mathrm{SO})}$ in $\mathrm{Xe}^{+}$ is $3.2 \mathrm{fs}$ [91]. In order to facilitate the comparison between $\mathrm{Ne}^{+}$and $\mathrm{Xe}^{+}$, we have introduced in Figs. 3 and 4 the phase

$$
\chi=2 \pi \frac{t-t_{\mathrm{end}}}{T^{(\mathrm{SO})}}
$$

where $t_{\text {end }}$ is the time at which the laser pulse ends. Hence, $\chi=2 \pi$, for example, indicates the completion of a full spinorbit cycle after the end of the laser pulse. 


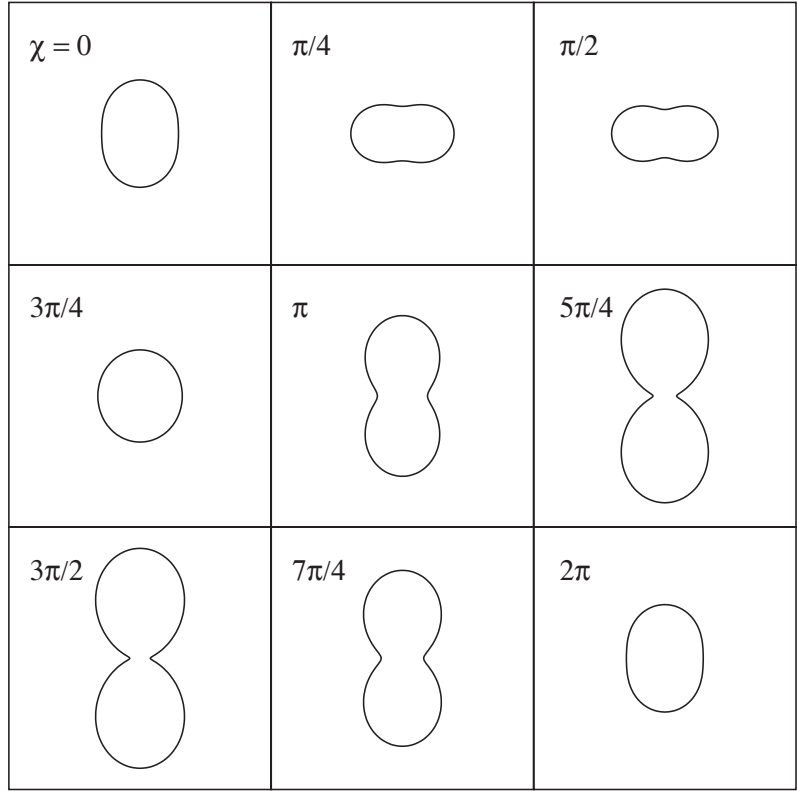

FIG. 3. Time evolution of the hole density of laser-generated $\mathrm{Ne}^{+}$. Polar plots are shown for nine different time delays after the end of the laser pulse.

A comparison between Figs. 3 and 4 shows clearly that the hole dynamics is much more pronounced for $\mathrm{Ne}^{+}$than for $\mathrm{Xe}^{+} . \mathrm{Ne}^{+}$evolves from an oval, prolate shape $(\chi=0)$ into an oblate shape $(\chi=\pi / 4$ and $\chi=\pi / 2)$. Later, at $\chi=5 \pi / 4$ and $\chi=3 \pi / 2$, the hole density of $\mathrm{Ne}^{+}$resembles the density one would obtain for a pure $2 p_{z}$ hole. In contrast, the hole density of $\mathrm{Xe}^{+}$has a peanutlike shape throughout. The hole density of $\mathrm{Xe}^{+}$undergoes relatively weak modulations as a function of time. This is a direct consequence of the suppressed coherence in laser-generated $\mathrm{Xe}^{+}$.

\section{CONCLUSIONS}

We have developed a theoretical description of multichannel strong-field ionization. Within this description, we determined the reduced density matrix of the laser-generated ions. We analyzed the time-dependent hole density and expressed it in terms of the ion density matrix. Extensive wave-packet calculations on neon and xenon under matched field amplitude conditions reveal that the quantum-state populations in the two noble-gas species are qualitatively similar, whereas the coherences are substantially different.

In order to populate the ionization channels in a coherent fashion, the time scale on which the channel wave packets in the $j=3 / 2$ and $j=1 / 2$ channels are formed has to be short in comparison to the spin-orbit period. In $\mathrm{Ne}$, this criterion is approximately satisfied. Ionization of $\mathrm{Ne}$ is saturated before significant spin-orbit dynamics occurs. The two spin-orbit channels are thus populated in phase. Effectively, this leads to the periodic formation of a $\mathrm{Ne}^{+}$state that is close to a pure $2 p_{z}$ hole.

The spin-orbit period of Xe, however, is comparable to the period of an optical cycle. In particular, even at the high field amplitude assumed here, it takes several field cycles to

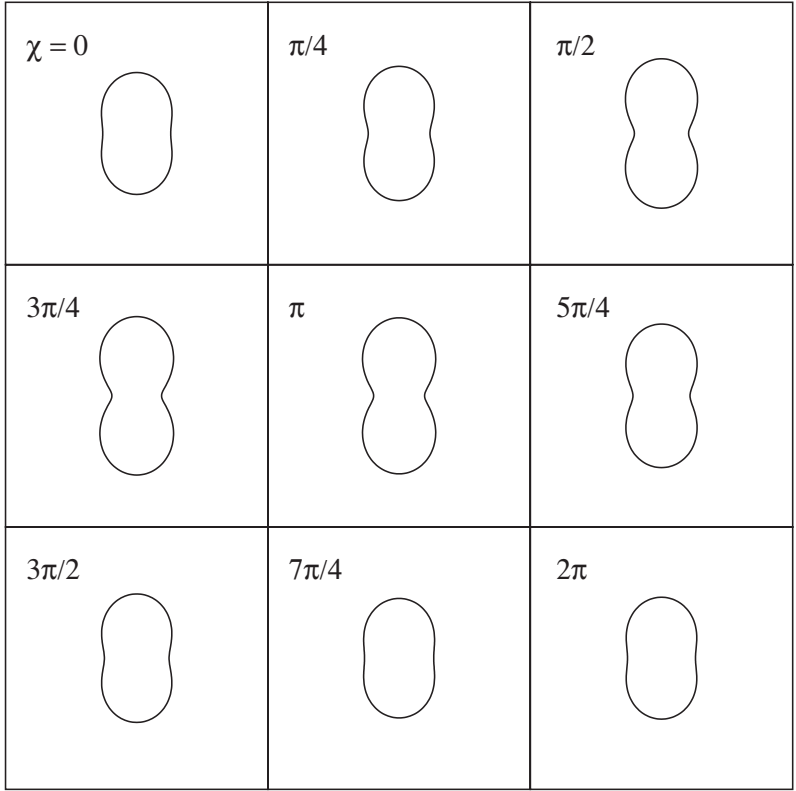

FIG. 4. Time evolution of the hole density of laser-generated $\mathrm{Xe}^{+}$. Polar plots are shown for nine different time delays after the end of the laser pulse.

completely saturate ionization. Hence, the amplitude added to the ionization channels during each half cycle is generally not in phase with the channel wave functions populated in previous half cycles. In order to create a more coherent electronic wave packet in the outer valence shell of $\mathrm{Xe}^{+}$, one could, in principle, ionize Xe using a series of ultrashort electric field spikes that are temporally separated by the spinorbit period of $3.2 \mathrm{fs}$. This seems difficult to implement in an experiment. On the other hand, if one used one or more extreme-ultraviolet attosecond pulses, one would not address primarily the outer valence shell.

Considerations similar to those presented here are probably applicable to strong-field ionization of molecules as well. The impact of multiple electronic channels on highharmonic generation by molecules has been the subject of recent studies $[122,123]$. If the binding energies associated with the channels populated in the strong-field ionization process are separated by approximately $1.55 \mathrm{eV}(800 \mathrm{~nm})$ or more, it is likely that the electronic density matrix of strongfield-generated molecular ions is characterized by an incoherent superposition of electronic states. This might change, however, if the laser electric field can directly couple the electronic states of the molecular ion.

\section{ACKNOWLEDGMENTS}

We thank Zhi-Heng Loh, Stephen R. Leone, Eleftherios Goulielmakis, and Ferenc Krausz for discussions. Part of this work was performed under the auspices of the U.S. Department of Energy by Lawrence Livermore National Laboratory under Contract No. DE-AC52-07NA27344. R.S. was supported by the Office of Basic Energy Sciences, Office of Science, U.S. Department of Energy, under Contract No. DE-AC02-06CH11357. 
[1] L. V. Keldysh, Zh. Eksp. Teor. Fiz. 47, 1945 (1964) [Sov. Phys. JETP 20, 1307 (1965)].

[2] F. H. M. Faisal, J. Phys. B 6, L89 (1973).

[3] L. A. Lompre, G. Mainfray, C. Manus, S. Repoux, and J. Thebault, Phys. Rev. Lett. 36, 949 (1976).

[4] H. R. Reiss, Phys. Rev. A 22, 1786 (1980).

[5] P. Lambropoulos, Phys. Rev. Lett. 55, 2141 (1985).

[6] M. V. Ammosov, N. B. Delone, and V. P. Krainov, Zh. Eksp. Teor. Fiz. 91, 2008 (1986) [Sov. Phys. JETP 64, 1191 (1986)].

[7] F. Yergeau, S. L. Chin, and P. Lavigne, J. Phys. B 20, 723 (1987).

[8] S. Augst, D. Strickland, D. D. Meyerhofer, S. L. Chin, and J. H. Eberly, Phys. Rev. Lett. 63, 2212 (1989).

[9] T. E. Glover, J. K. Crane, M. D. Perry, R. W. Lee, and R. W. Falcone, Phys. Rev. Lett. 75, 445 (1995).

[10] G. N. Gibson, M. Li, C. Guo, and J. Neira, Phys. Rev. Lett. 79, 2022 (1997)

[11] J. Muth-Böhm, A. Becker, and F. H. M. Faisal, Phys. Rev. Lett. 85, 2280 (2000).

[12] R. Taïeb, V. Véniard, and A. Maquet, Phys. Rev. Lett. 87, 053002 (2001).

[13] R. Moshammer, J. Ullrich, B. Feuerstein, D. Fischer, A. Dorn, C. D. Schröter, J. R. Crespo Lopez-Urrutia, C. Hoehr, H. Rottke, C. Trump, M. Wittmann, G. Korn, and W. Sandner, Phys. Rev. Lett. 91, 113002 (2003).

[14] E. Gubbini, U. Eichmann, M. Kalashnikov, and W. Sandner, Phys. Rev. Lett. 94, 053602 (2005).

[15] O. Smirnova, M. Spanner, and M. Ivanov, Phys. Rev. A 77, 033407 (2008).

[16] P. Eckle, A. N. Pfeiffer, C. Cirelli, A. Staudte, R. Dörner, H. G. Muller, M. Büttiker, and U. Keller, Science 322, 1525 (2008).

[17] P. Agostini, F. Fabre, G. Mainfray, G. Petite, and N. K. Rahman, Phys. Rev. Lett. 42, 1127 (1979).

[18] R. R. Freeman, P. H. Bucksbaum, H. Milchberg, S. Darack, D. Schumacher, and M. E. Geusic, Phys. Rev. Lett. 59, 1092 (1987).

[19] P. B. Corkum, N. H. Burnett, and F. Brunel, Phys. Rev. Lett. 62, 1259 (1989).

[20] E. Mevel, P. Breger, R. Trainham, G. Petite, P. Agostini, J. P. Chambaret, A. Migus, and A. Antonetti, J. Phys. B 25, L401 (1992).

[21] E. Mevel, P. Breger, R. Trainham, G. Petite, P. Agostini, A. Migus, J. P. Chambaret, and A. Antonetti, Phys. Rev. Lett. 70, 406 (1993).

[22] U. Mohideen, M. H. Sher, H. W. K. Tom, G. D. Aumiller, O. R. Wood, R. R. Freeman, J. Bokor, and P. H. Bucksbaum, Phys. Rev. Lett. 71, 509 (1993).

[23] G. G. Paulus, F. Zacher, H. Walther, A. Lohr, W. Becker, and M. Kleber, Phys. Rev. Lett. 80, 484 (1998).

[24] T. Morishita, A.-T. Le, Z. Chen, and C. D. Lin, Phys. Rev. Lett. 100, 013903 (2008).

[25] M. Okunishi, T. Morishita, G. Prümper, K. Shimada, C. D. Lin, S. Watanabe, and K. Ueda, Phys. Rev. Lett. 100, 143001 (2008).

[26] M. Busuladzic, A. Gazibegovic-Busuladzic, D. B. Milosevic, and W. Becker, Phys. Rev. Lett. 100, 203003 (2008).

[27] B. Walker, B. Sheehy, L. F. DiMauro, P. Agostini, K. J. Schafer, and K. C. Kulander, Phys. Rev. Lett. 73, 1227 (1994).

[28] Th. Weber, H. Giessen, M. Weckenbrock, G. Urbasch, A. Staudte, L. Spielberger, O. Jagutzki, V. Mergel, M. Vollmer, and R. Dörner, Nature (London) 405, 658 (2000).

[29] R. Moshammer, B. Feuerstein, W. Schmitt, A. Dorn, C. D. Schröter, J. Ullrich, H. Rottke, C. Trump, M. Wittmann, G. Korn, K. Hoffmann, and W. Sandner, Phys. Rev. Lett. 84, 447 (2000).

[30] A. Becker and F. H. M. Faisal, Phys. Rev. Lett. 84, 3546 (2000).

[31] R. Kopold, W. Becker, H. Rottke, and W. Sandner, Phys. Rev. Lett. 85, 3781 (2000).

[32] A. S. Alnaser, X. M. Tong, T. Osipov, S. Voss, C. M. Maharjan, P. Ranitovic, B. Ulrich, B. Shan, Z. Chang, C. D. Lin, and C. L. Cocke, Phys. Rev. Lett. 93, 183202 (2004).

[33] P. J. Ho, R. Panfili, S. L. Haan, and J. H. Eberly, Phys. Rev. Lett. 94, 093002 (2005).

[34] S. Gräfe and M. Yu. Ivanov, Phys. Rev. Lett. 99, 163603 (2007).

[35] P. Koval, F. Wilken, D. Bauer, and C. H. Keitel, Phys. Rev. Lett. 98, 043904 (2007).

[36] A. Rudenko, V. L. B. de Jesus, Th. Ergler, K. Zrost, B. Feuerstein, C. D. Schröter, R. Moshammer, and J. Ullrich, Phys. Rev. Lett. 99, 263003 (2007).

[37] A. McPherson, G. Gibson, H. Jara, U. Johann, T. S. Luk, I. A. McIntyre, K. Boyer, and C. K. Rhodes, J. Opt. Soc. Am. B 4, 595 (1987).

[38] M. Ferray, A. L'Huillier, X. F. Li, L. A. Lompre, G. Mainfray, and C. Manus, J. Phys. B 21, L31 (1988).

[39] A. L'Huillier, K. J. Schafer, and K. C. Kulander, Phys. Rev. Lett. 66, 2200 (1991)

[40] P. B. Corkum, Phys. Rev. Lett. 71, 1994 (1993).

[41] M. Lewenstein, Ph. Balcou, M. Yu. Ivanov, A. L'Huillier, and P. B. Corkum, Phys. Rev. A 49, 2117 (1994).

[42] Ch. Spielmann, N. H. Burnett, S. Santania, R. Koppitsch, M. Schnürer, C. Kan, M. Lenzner, P. Wobrauschek, and F. Krausz, Science 278, 661 (1997).

[43] Z. Chang, A. Rundquist, H. Wang, M. M. Murnane, and H. C. Kapteyn, Phys. Rev. Lett. 79, 2967 (1997).

[44] E. A. Gibson, A. Paul, N. Wagner, R. Tobey, D. Gaudiosi, S. Backus, I. P. Christov, A. Aquila, E. M. Gullikson, D. T. Attwood, M. M. Murnane, and H. C. Kapteyn, Science 302, 95 (2003).

[45] E. A. Gibson, A. Paul, N. Wagner, R. Tobey, S. Backus, I. P. Christov, M. M. Murnane, and H. C. Kapteyn, Phys. Rev. Lett. 92, 033001 (2004).

[46] J. Seres, E. Seres, A. J. Verhoef, G. Tempea, C. Streli, P. Wobrauschek, V. Yakovlev, A. Scrinzi, C. Spielmann, and F. Krausz, Nature (London) 433, 596 (2005).

[47] A. Gordon and F. X. Kärtner, Phys. Rev. Lett. 95, 223901 (2005).

[48] A. Gordon, F. X. Kärtner, N. Rohringer, and R. Santra, Phys. Rev. Lett. 96, 223902 (2006).

[49] J. Levesque, D. Zeidler, J. P. Marangos, P. B. Corkum, and D. M. Villeneuve, Phys. Rev. Lett. 98, 183903 (2007).

[50] M. Spanner and P. Brumer, Phys. Rev. A 78, 033840 (2008).

[51] G. Jordan and A. Scrinzi, New J. Phys. 10, 025035 (2008).

[52] M. Hentschel, R. Kienberger, Ch. Spielmann, G. A. Reider, N. Milosevic, T. Brabec, P. Corkum, U. Heinzmann, M. Drescher, and F. Krausz, Nature (London) 414, 509 (2001).

[53] M. Drescher, M. Hentschel, R. Kienberger, M. Ulberacker, V. Yakovlev, A. Scrinzi, T. Westerwalbesloh, U. Kleineberg, U. Heinzmann, and F. Krausz, Nature (London) 419, 803 (2002). 
[54] P. Agostini and L. F. DiMauro, Rep. Prog. Phys. 67, 813 (2004).

[55] F. Lindner, M. G. Schätzel, H. Walther, A. Baltuska, E. Goulielmakis, F. Krausz, D. B. Milošević, D. Bauer, W. Becker, and G. G. Paulus, Phys. Rev. Lett. 95, 040401 (2005).

[56] G. L. Yudin, A. D. Bandrauk, and P. B. Corkum, Phys. Rev. Lett. 96, 063002 (2006).

[57] T. Pfeifer, M. J. Abel, P. M. Nagel, A. Jullien, Z.-H. Loh, M. J. Bell, D. M. Neumark, and S. R. Leone, Chem. Phys. Lett. 463, 11 (2008).

[58] A. D. Bandrauk, S. Chelkowski, S. Kawai, and H. Lu, Phys. Rev. Lett. 101, 153901 (2008).

[59] R. Velotta, N. Hay, M. B. Mason, M. Castillejo, and J. P. Marangos, Phys. Rev. Lett. 87, 183901 (2001).

[60] H. Niikura, F. Légaré, R. Hasbani, A. D. Bandrauk, M. Yu. Ivanov, D. M. Villeneuve, and P. B. Corkum, Nature (London) 417, 917 (2002).

[61] J. Itatani, J. Levesque, D. Zeidler, H. Niikura, H. Pépin, J. C. Kieffer, P. B. Corkum, and D. M. Villeneuve, Nature (London) 432, 867 (2004).

[62] J. Itatani, D. Zeidler, J. Levesque, M. Spanner, D. M. Villeneuve, and P. B. Corkum, Phys. Rev. Lett. 94, 123902 (2005).

[63] Th. Ergler, A. Rudenko, B. Feuerstein, K. Zrost, C. D. Schröter, R. Moshammer, and J. Ullrich, Phys. Rev. Lett. 95, 093001 (2005).

[64] R. Santra and A. Gordon, Phys. Rev. Lett. 96, 073906 (2006).

[65] S. Patchkovskii, Z. Zhao, T. Brabec, and D. M. Villeneuve, Phys. Rev. Lett. 97, 123003 (2006).

[66] B. D. Esry, A. M. Sayler, P. Q. Wang, K. D. Carnes, and I. Ben-Itzhak, Phys. Rev. Lett. 97, 013003 (2006).

[67] N. L. Wagner, A. Wüest, I. P. Christov, T. Popmintchev, X. Zhou, M. M. Murnane, and H. C. Kapteyn, Proc. Natl. Acad. Sci. U.S.A. 103, 13279 (2006).

[68] S. Baker, J. S. Robinson, C. A. Haworth, H. Teng, R. A. Smith, C. C. Chirila, M. Lein, J. W. G. Tisch, and J. P. Marangos, Science 312, 424 (2006).

[69] M. Lein, J. Phys. B 40, R135 (2007).

[70] O. Smirnova, A. S. Mouritzen, S. Patchkovskii, and M. Yu. Ivanov, J. Phys. B 40, F197 (2007).

[71] Z. Zhao, J. Yuan, and T. Brabec, Phys. Rev. A 76, 031404(R) (2007).

[72] S. Patchkovskii, Z. Zhao, T. Brabec, and D. M. Villeneuve, J. Chem. Phys. 126, 114306 (2007).

[73] B. Feuerstein, Th. Ergler, A. Rudenko, K. Zrost, C. D. Schröter, R. Moshammer, J. Ullrich, T. Niederhausen, and U. Thumm, Phys. Rev. Lett. 99, 153002 (2007).

[74] E. V. van der Zwan, C. C. Chirila, and M. Lein, Phys. Rev. A 78, 033410 (2008).

[75] Z. B. Walters, S. Tonzani, and C. H. Greene, J. Phys. Chem. A 112, 9439 (2008).

[76] W. Li, X. Zhou, R. Lock, S. Patchkovskii, A. Stolow, H. C. Kapteyn, and M. M. Murnane, Science 322, 1207 (2008).

[77] K. C. Kulander, Phys. Rev. A 38, 778 (1988).

[78] J. Javanainen, J. H. Eberly, and Q. Su, Phys. Rev. A 38, 3430 (1988).

[79] K. C. Kulander and B. W. Shore, Phys. Rev. Lett. 62, 524 (1989).

[80] J. H. Eberly, Q. Su, and J. Javanainen, Phys. Rev. Lett. 62, 881 (1989).

[81] W. Becker, S. Long, and J. K. McIver, Phys. Rev. A 41, 4112
(1990).

[82] W. Becker, S. Long, and J. K. McIver, Phys. Rev. A 50, 1540 (1994).

[83] N. H. Shon, A. Suda, and K. Midorikawa, Phys. Rev. A 62, 023801 (2000)

[84] M. Awasthi, Y. V. Vanne, A. Saenz, A. Castro, and P. Decleva, Phys. Rev. A 77, 063403 (2008).

[85] H. Rottke, J. Ludwig, and W. Sandner, J. Phys. B 29, 1479 (1996).

[86] P. Kaminski, R. Wiehle, V. Renard, A. Kazmierczak, B. Lavorel, O. Faucher, and B. Witzel, Phys. Rev. A 70, 053413 (2004).

[87] L. Young, D. A. Arms, E. M. Dufresne, R. W. Dunford, D. L. Ederer, C. Höhr, E. P. Kanter, B. Krässig, E. C. Landahl, E. R. Peterson, J. Rudati, R. Santra, and S. H. Southworth, Phys. Rev. Lett. 97, 083601 (2006).

[88] C. Höhr, E. R. Peterson, N. Rohringer, J. Rudati, D. A. Arms, E. M. Dufresne, R. W. Dunford, D. L. Ederer, E. P. Kanter, B. Krässig, E. C. Landahl, R. Santra, S. H. Southworth, and L. Young, Phys. Rev. A 75, 011403(R) (2007).

[89] Z.-H. Loh, M. Khalil, R. E. Correa, R. Santra, C. Buth, and S. R. Leone, Phys. Rev. Lett. 98, 143601 (2007).

[90] S. H. Southworth, D. A. Arms, E. M. Dufresne, R. W. Dunford, D. L. Ederer, C. Höhr, E. P. Kanter, B. Krässig, E. C. Landahl, E. R. Peterson, J. Rudati, R. Santra, D. A. Walko, and L. Young, Phys. Rev. A 76, 043421 (2007).

[91] R. Santra, R. W. Dunford, and L. Young, Phys. Rev. A 74, 043403 (2006).

[92] N. Rohringer, A. Gordon, and R. Santra, Phys. Rev. A 74, 043420 (2006).

[93] J. Breidbach and L. S. Cederbaum, Phys. Rev. Lett. 94, 033901 (2005).

[94] A. I. Kuleff, J. Breidbach, and L. S. Cederbaum, J. Chem. Phys. 123, 044111 (2005).

[95] A. L. Fetter and J. D. Walecka, Quantum Theory of ManyParticle Systems (McGraw-Hill, Boston, 1971).

[96] A. A. Abrikosov, L. P. Gorkov, and I. E. Dzyaloshinski, Methods of Quantum Field Theory in Statistical Physics (Dover, New York, 1975).

[97] A. Goldberg and B. W. Shore, J. Phys. B 11, 3339 (1978).

[98] G. Jolicard and E. J. Austin, Chem. Phys. Lett. 121, 106 (1985).

[99] U. V. Riss and H.-D. Meyer, J. Phys. B 26, 4503 (1993).

[100] R. Santra and L. S. Cederbaum, Phys. Rep. 368, 1 (2002).

[101] J. G. Muga, J. P. Palao, B. Navarro, and I. L. Egusquiza, Phys. Rep. 395, 357 (2004).

[102] A. Szabo and N. S. Ostlund, Modern Quantum Chemistry (Dover, Mineola, New York, 1996).

[103] K. Blum, Density Matrix Theory and Applications (Plenum, New York, 1996).

[104] J. C. Slater, Phys. Rev. 81, 385 (1951).

[105] J. C. Slater and K. H. Johnson, Phys. Rev. B 5, 844 (1972).

[106] F. Herman and S. Skillman, Atomic Structure Calculations (Prentice-Hall, Englewood Cliffs, NJ, 1963).

[107] J. W. Cooper, Phys. Rev. 128, 681 (1962).

[108] S. T. Manson and J. W. Cooper, Phys. Rev. 165, 126 (1968).

[109] J. J. Yeh and I. Lindau, At. Data Nucl. Data Tables 32, 1 (1985).

[110] R. Santra and C. H. Greene, Phys. Rev. A 70, 053401 (2004).

[111] P. Strange, Relativistic Quantum Mechanics (Cambridge Uni- 
versity Press, Cambridge, England, 1998).

[112] E. U. Condon and G. H. Shortley, The Theory of Atomic Spectra (Cambridge University Press, Cambridge, England, 1951).

[113] M. E. Rose, Elementary Theory of Angular Momentum (Dover, New York, 1995).

[114] J. Wang, Shih-I Chu, and C. Laughlin, Phys. Rev. A 50, 3208 (1994).

[115] G. H. Yao and S. I. Chu, Chem. Phys. Lett. 204, 381 (1993).

[116] X. M. Tong and S. I. Chu, Chem. Phys. 217, 119 (1997).

[117] B. Fornberg, A Practical Guide to Pseudospectral Methods (Cambridge University Press, Cambridge, England, 1996).
[118] V. Kaufman and L. Minnhagen, J. Opt. Soc. Am. 62, 92 (1972).

[119] F. Brandi, I. Velchev, W. Hogervorst, and W. Ubachs, Phys. Rev. A 64, 032505 (2001).

[120] W. Persson, Phys. Scr. 3, 133 (1971).

[121] J. E. Hansen and W. Persson, Phys. Scr. 36, 602 (1987).

[122] B. K. McFarland, J. P. Farrell, P. H. Bucksbaum, and M. Gühr, Science 322, 1232 (2008).

[123] O. Smirnova, S. Patchkovskii, Y. Mairesse, N. Dudovich, D. Villeneuve, P. Corkum, and M. Yu. Ivanov, Phys. Rev. Lett. 102, 063601 (2009). 